\title{
An Improved Lumped Parameter Model for a Piezoelectric Energy Harvester in Transverse Vibration
}

\author{
Guang-qing Wang and Yue-ming Lu \\ School of Information \& Electronics Engineering, Zhejiang Gongshang University, No. 18 Xuezheng Street, \\ Xiasha University Town, Hangzhou 310018, China \\ Correspondence should be addressed to Guang-qing Wang; kele76@163.com
}

Received 26 February 2013; Accepted 20 June 2013; Published 24 February 2014

Academic Editor: Hassan Haddadpour

Copyright ( 2014 G.-q. Wang and Y.-m. Lu. This is an open access article distributed under the Creative Commons Attribution License, which permits unrestricted use, distribution, and reproduction in any medium, provided the original work is properly cited.

\begin{abstract}
An improved lumped parameter model (ILPM) is proposed which predicts the output characteristics of a piezoelectric vibration energy harvester (PVEH). A correction factor is derived for improving the precisions of lumped parameter models for transverse vibration, by considering the dynamic mode shape and the strain distribution of the PVEH. For a tip mass, variations of the correction factor with PVEH length are presented with curve fitting from numerical solutions. The improved governing motion equations and exact analytical solution of the PVEH excited by persistent base motions are developed. Steady-state electrical and mechanical response expressions are derived for arbitrary frequency excitations. Effects of the structural parameters on the electromechanical outputs of the PVEH and important characteristics of the PVEH, such as short-circuit and open-circuit behaviors, are analyzed numerically in detail. Accuracy of the output performances of the ILPM is identified from the available lumped parameter models and the coupled distributed parameter model. Good agreement is found between the analytical results of the ILPM and the coupled distributed parameter model. The results demonstrate the feasibility of the ILPM as a simple and effective means for enhancing the predictions of the PVEH.
\end{abstract}

\section{Introduction}

Wireless sensors and communication node networks have recently been attracting a large amount of interest because of their rapid development and wide applications [1, 2]. However, as the networks increase in number and the devices decrease in size, the proliferation of these autonomous microsensors raises the problem of effective power supply. Batteries used to power them may not only increase the size and weight of the devices but also suffer from a brief service life, presenting major limitations to system lifetime [3-5]. However, harvesting energy through the direct piezoelectric effect by employing the ambient vibration of the devices offers promising solutions [6-8].

Research into piezoelectric vibration energy harvesters (PVEH) involves understanding the mechanics of vibrating structures, the constitutive behavior of piezoelectric materials, and elementary circuit theory. Some researchers have focused on the mathematical modeling of the PVEH.
An accurate mathematical model can be used to predict the electrical outputs and to optimize the PVEH to obtain the maximum electrical output for a given input. Existing approaches to modeling PVEH include single-degreeof-freedom (SDOF) models (or lumped parameter models $(\mathrm{LPM})$ ) $[7,9,10]$, approximate distributed parameter models with the Rayleigh-Ritz method [11, 12], equivalent circuit models, and other distributed parameter modeling approaches [13-16].

As described by Roundy et al. [9] and duToit et al. [17], the SDOF modeling approach treats the PVEH as a massspring-damper system, which is convenient for coupling the mechanical part of the PVEH with a simple electrical circuit. Although the SDOF model enables initial investigation into the problem by allowing simple closed-form expressions, it lacks several important aspects of the physical system, such as dynamic mode and accurate strain distribution along the PVEH, as well as their effects on the electrical response. Because the PVEH is basically excited by the motion of 


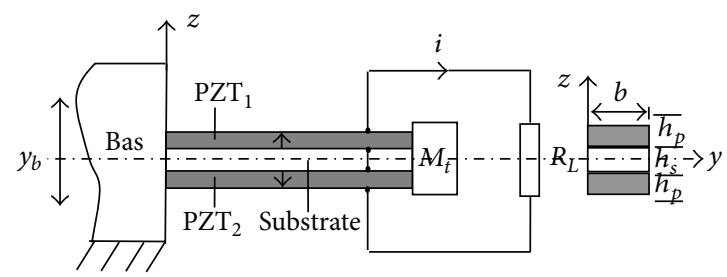

FIGURE 1: Cantilevered piezoelectric energy harvester excited by the motion of its base in the transverse direction.

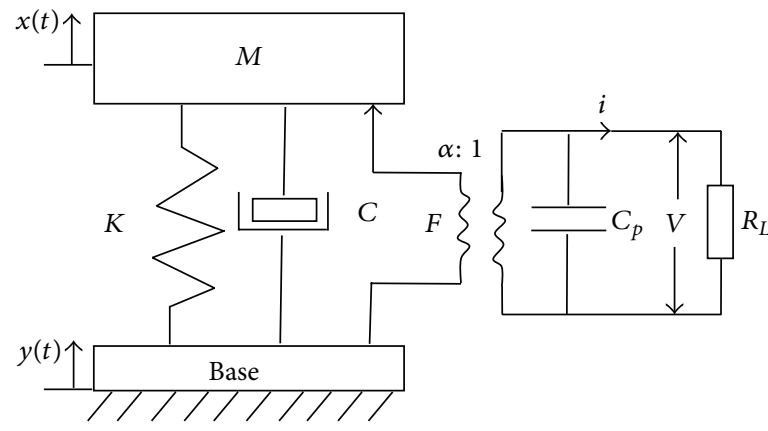

Figure 2: Coupled LPM of the PVEH.

the base, the SDOF harmonic base excitation relation has been used in PVEH for both modeling and studying the maximum power outputs and parameter optimization [17-19]. It was recently shown that the SODF model may produce highly inaccurate results for both transverse vibrations and longitudinal vibrations [13-15]. This is because the contribution of the distributed mass of the cantilevered beam to the excitation amplitude, which is not modeled by Roundy et al. [9], is underestimated because of the SDOF in a work by duToit et al. [17]. Erturk and Inman [13-15] investigated existing problems in the commonly used SDOF model of the PVEH and give a correct representation of the SDOF model by treating the air damping and structural damping separately. They assumed that the air damping coefficient is proportional to the equivalent mass, whereas the structural damping coefficient is proportional to the equivalent stiffness. Erturk and Inman [13-15] also give expressions of the relative tip response, obtained from SDOF and Euler-Bernoulli models. By comparing these two models, an amplitude correction factor was derived to improve the prediction of the SDOF model. Their results show that the error in predicting the fundamental natural frequency of the PVEH is approximately $0.5 \%$ in the absence of a tip mass relative to the EulerBernoulli model fundamental natural frequency. However, the relative tip response of the PVEH is difficult and more complicated to obtain with the Euler-Bernoulli assumption.

Unlike the above-mentioned studies, this paper presents an improved lumped parameter model (ILPM) in which the electromechanical coupled effects as well as the dynamic mode and the accurate strain distribution of the PVEH are taken into account. Based on the Rayleigh energy method, a parameter (not amplitude) correction factor is derived for improving the precisions of the respective LPMs for transverse vibration. Variations of the correction factor with harvester length are presented graphically along with curve fitting obtained from numerical solutions. Because the ILPM used only the shape mode function and the static deflection of the PVEH, and not the tip relative response, it is easy to define the model parameters. The presented ILPM provides a simple and effective approach to modeling and predicting the PVEH in transverse vibrations.

\section{Improved Lumped Parameter Model}

Figure 1 shows the structure of the PVEH, which consists of a cantilevered bimorph beam with tip mass $M_{t}$. Two piezoelectric plates $\left(\mathrm{PZT}_{1}\right.$ and $\left.\mathrm{PZT}_{2}\right)$ oppositely polarized in the thickness direction are perfectly bonded to the top and bottom, respectively, of the substrate beam. The piezoelectric bimorphs are connected in series. The electrode pairs covering the top and bottom faces of the piezoelectric plates are assumed to be thin so that their contribution to the thickness dimension is negligible. A simple electrical circuit consisting of a resistive load $\left(R_{L}\right)$ is directly connected to the output terminal of the harvester. One end of the piezoelectric cantilever is fixed to the base. We assume persistent excitation at the base of the harvester so that continuous electrical outputs can be extracted from the resistive load.

As described by Roundy et al. [9] and duToit et al. [17], the PVEH shown in Figure 1 can be treated as a SDOF system consisting of a mass-spring-damper system, as shown in Figure 2. The equivalent mass, damping coefficient, and equivalent stiffness are denoted by $M, C$, and $K$, respectively. The PZT element is characterized by an electromechanical coupled factor $\alpha$ and electrical capacitance $C_{p}$, and $y(t)$ and $x(t)$ denote the displacements of the base vibration and the equivalent mass $M$, respectively. 
According to the method used to obtain the differential equation, the governing equation of motion due to the vibrating base can be written as $[20,21]$

$$
\begin{gathered}
M \ddot{x}(t)+C \dot{x}(t)+K x(t)+\alpha V(t)=C \dot{y}(t)+K y(t), \\
\alpha[\dot{x}(t)-\dot{y}(t)]-C_{p} \dot{V}(t)=i(t)=\frac{V(t)}{R_{L}},
\end{gathered}
$$

where $i(t)$ and $V(t)$ are the current and voltage of the load resistance, respectively, as shown in Figure 2. If the base displacement is harmonic in the form of $y(t)=Y_{0} e^{j \omega t}$ (where $Y_{0}$ is the displacement amplitude, $\omega$ is the frequency, and $j$ is the unit imaginary number), then the steady-state response of the lumped mass relative to the base can be expressed as

$$
\begin{aligned}
& x(t) \\
& =\frac{j 2 \xi_{n} \omega_{n} \omega+\omega_{n}^{2}+\left(j \omega \alpha^{2} / M\left(j \omega C_{p}+1 / R_{L}\right)\right)}{j 2 \xi_{n} \omega_{n} \omega+\omega_{n}^{2}-\omega^{2}+\left(j \omega \alpha^{2} / M\left(j \omega C_{p}+1 / R_{L}\right)\right)}, \\
& \quad \times Y_{0} e^{j \omega t},
\end{aligned}
$$

where $\omega_{n}=\sqrt{K / M}, 2 \xi_{n}=C / \sqrt{K M}$.

Substituting (3) into (2), the steady-state response of the load resistance voltage relative to the base can be obtained:

$$
\begin{aligned}
& V(t) \\
& =\frac{j \omega \alpha}{j \omega C_{p}+1 / R_{L}} \\
& \quad \cdot \frac{\omega^{2}}{j 2 \xi_{n} \omega_{n} \omega+\omega_{n}^{2}-\omega^{2}+\left(j \omega \alpha^{2} / M\left(j \omega C_{p}+1 / R_{L}\right)\right)} \\
& \quad \times Y_{0} e^{j \omega t} .
\end{aligned}
$$

Rearranging (3) and (4), the steady-state velocity response of the lumped mass and the voltage response of the load resistance relative to the base vibrating acceleration can be given by

$$
\begin{aligned}
& \frac{\dot{x}(t)}{\ddot{y}(t)} \\
& =\frac{1}{j \omega}+\frac{-j \omega\left(j \omega C_{p} R_{L}+1\right) M}{M\left(j 2 \xi_{n} \omega_{n} \omega+\omega_{n}^{2}-\omega^{2}\right)\left(j \omega C_{p} R_{L}+1\right)+j \omega R_{L} \alpha^{2}},
\end{aligned}
$$

$$
\frac{V(t)}{\ddot{y}(t)}=\frac{-j \omega \alpha R_{L} M}{M\left(j 2 \xi_{n} \omega_{n} \omega+\omega_{n}^{2}-\omega^{2}\right)\left(j \omega C_{p} R_{L}+1\right)+j \omega \alpha^{2} R_{L}} .
$$

The output power of the load resistance relative to the base vibrating acceleration can be obtained from (6) as

$$
\begin{aligned}
& \frac{P(t)}{\ddot{y}^{2}(t)} \\
& =\frac{1}{R_{L}} \cdot \frac{V^{2}(t)}{\ddot{y}^{2}(t)} \\
& =\frac{1}{R_{L}} \cdot\left(\frac{-j \omega \alpha R_{L} M}{M\left(j 2 \xi_{n} \omega_{n} \omega+\omega_{n}^{2}-\omega^{2}\right)\left(j \omega C_{p} R_{L}+1\right)+j \omega \alpha^{2} R_{L}}\right)^{2} .
\end{aligned}
$$

\section{Definition of the Model Parameters}

3.1. Equivalent Parameters of the Available LPM. The LPMs have two important lumped parameters: the equivalent mass $M$ and the equivalent stiffness $K$. These two parameters are dependent on the material constants and the design of the PVEH and can be derived with standard modal analysis [2, 15, 19]. Typically, $K$ is obtained from the static deflection relation of a cantilevered beam to a concentrated transverse load at the tip, and $M$ is obtained by expressing the total kinetic energy of the beam in terms of the velocity at the tip.

Because vibration-based energy harvesters are excited by the motion of their base, many researchers have used the uncoupled lumped parameter model (ULPM) of base excitation $[1,9,10,19]$. The equivalent parameters of the ULPM presented by Williams and Yates [10] are described by

$$
M=\frac{\rho A L}{3}+M_{t}, \quad K=\frac{3 E I}{L^{3}},
$$

where $E I$ is the equivalent bending stiffness of the cantilevered beam, and

$$
\begin{gathered}
I=\frac{2 b\left[\left(h_{p}+h_{s} / 2\right)^{3}-\left(h_{s} / 2\right)^{3}\right]}{3}+\frac{b h_{s}^{3}}{12}, \\
E=\frac{E_{s}\left(b h_{s}^{3} / 12\right)+E_{p}\left(2 b\left[\left(h_{p}+h_{s} / 2\right)^{3}-\left(h_{s} / 2\right)^{3}\right] / 3\right)}{I}, \\
\rho=\frac{2 \rho_{p} h_{p}+\rho_{s} h_{s}}{2 h_{p}+h_{s}}, \quad A=b\left(h_{s}+2 h_{p}\right),
\end{gathered}
$$

where $\rho_{s}$ and $\rho_{p}$ are the density of the substrate beam and PZT, respectively; $L$ and $b$ are the axial length and width of the piezoelectric cantilever, respectively; $h_{s}$ and $h_{p}, E_{s}$ and $E_{p}$, and $\rho_{s}$ and $\rho_{p}$ are the thickness, stiffness, and density of the substrate beam and PZT, respectively.

Erturk and Inman [13-15] point out that using equivalent parameters of (8) of the ULPM to predict the relative motion at the tip of the PVEH will result in underestimation with an error greater than 35\% regardless of the damping ratio. Because it does not model the effect of piezoelectric coupling in the PVEH, the ULPM is also unable to predict the frequency shift due to changing the load resistance [13-15]. 
To overcome these issues, Erturk and Inman [13-15] propose a coupled LPM. First, the distributed parameter solutions are presented with the relative tip response expressions of the PVEH obtained under the Euler-Bernoulli assumption, and then by comparing these relations with the ULPM solutions, an amplitude correction factor is introduced into the ULPM to improve its predictions. The lumped parameter relations of the LPM are defined as follows [13]:

$$
M=\frac{30 \rho A L}{144}+M_{t}, \quad K=\frac{3 E I}{L^{3}} .
$$

The results of Erturk and Inman's LPM show that the error due to using (10) in predicting the fundamental natural frequency is approximately $0.5 \%$ in the absence of a tip mass (relative to the Euler-Bernoulli model fundamental natural frequency). However, as mentioned in the Introduction, distributed parameter solutions and the relative tip response of the PVEH are difficult and more complicated to obtain with the Euler-Bernoulli assumption.

3.2. Definition of the Equivalent Parameters of ILPM. In this paper we present a simple and novel method to define the equivalent parameters of the PVEH with the curve-fitting method. The equivalent parameters of the PVEH are assumed to take the following forms:

$$
\begin{gathered}
M=\beta_{M} \rho A L+M_{t}, \\
K=\beta_{K} \frac{E I}{L^{3}},
\end{gathered}
$$

where $\beta_{M}$ and $\beta_{K}$ are the correction factors. For greater accuracy, information on the dynamic mode shape and strain distribution of the PVEH is considered. Steps for determining the parameters of $\beta_{M}$ and $\beta_{K}$ are based on the Rayleigh-Ritz approximation [22] and are as follows:

(i) structure an equation for the static bending deflection $w(x)$ of the PVEH;

(ii) solve the shape mode function $\phi(x)$ of the PVEH;

(iii) define the relationship between $w(x)$ and $\phi(x)$;

(iv) calculate the parameters correction factor $\mu(x)$ based on the relationship between $w(x)$ and $\phi(x)$;

(v) calculate the fitting result $\bar{\mu}(x)$ of $\mu(x)$ with the curvefitting method;

(vi) obtain the approximate solution of $w(x)$;

(vii) calculate the total kinetic energy of the ILPM and the ULPM, respectively;

(viii) define the equivalent mass $M$ according to energy conservation under the Rayleigh-Ritz approximation;

(ix) calculate the total potential energy of the ILPM and the ULPM, respectively;

(x) define the equivalent stiffness $K$ according to energy conservation under the Rayleigh-Ritz approximation.

We assume that $w(x)$ is the static bending deflection of the PVEH shown in Figure 1 and that the relationship between the deflection and the bending mode shape $\phi(x)$ of the clamped-free beam takes the form

$$
w(x)=\mu(x) \phi(x),
$$

where $\mu(x)$ is the parameter correction factor of the ILPM. The bending mode shape $\phi(x)$ of the clamped-free beam shown in Figure 1 can be given by [13]

$$
\phi(x)=C\left[\cos \frac{\lambda}{L} x-\cosh \frac{\lambda}{L} x+\sigma\left(\sin \frac{\lambda}{L} x-\sinh \frac{\lambda}{L} x\right)\right],
$$

where $\lambda=1.162$ and $C$ is a modal constant that can be calculated by normalizing the bending mode shape according to the following orthogonal condition [13]:

$$
\int_{0}^{L} \rho A \phi^{2}(x) d x+M_{t} \phi^{2}(L)+I_{t} \phi^{\prime 2}(L)=1,
$$

where $I_{t}$ is the rotary inertia of the tip mass $M_{t}$. Theoretically, the parameter correction factor $\mu(x)$ can be calculated from $[22,23]$

$$
\mu(x)=\frac{w(x)}{\phi(x)}=\frac{M_{t} g x^{2}(3 L-x)}{6 E I \phi(x)},
$$

where $g$ is gravitational acceleration. Here, the correction factor $\mu(x)$ can be determined by the curve-fitting method based on the theoretical results calculated from (16) with MATLAB software. The least-squares fitting method is adopted; the theoretical and fitting results are listed in Table 1. The fitting expression of the correction factor is obtained from the results of Table 1 [24]. Consider

$$
\bar{\mu}(x)=-\frac{2.7 \times 10^{-5}}{99 L}(x-0.01 L)-1.207 \times 10^{-5} .
$$

Figure 3 shows the theoretical and fitting results of the correction factor with the length of the cantilevered beam of the PVEH. It indicates that the fitting results show good agreement with those of the theoretical results, and the relative error between both results is less than $0.062 \%$. Therefore, in the following, we use $\bar{\mu}(x)$, instead of $\mu(x)$, to represent the correction factor.

Substituting (14) and (17) into (13), we can define the deflection $w(x)$ of the cantilevered beam. Then the kinetic energy and the potential energy of the PVEH without the tip mass $M_{t}$ are calculated as follows $[9,10,17]$ :

$$
\begin{gathered}
T=\frac{1}{2} \int_{0}^{L} \rho A d x \dot{w}^{2}(x)=\frac{1}{2} \int_{0}^{L} \rho A\left[\frac{\bar{\mu}(x) \phi(x)}{\bar{\mu}(L) \phi(L)}\right]^{2} d x \dot{w}^{2}(L), \\
E_{u}=\frac{1}{2} \int_{0}^{L}\left[\frac{\partial^{2} w(x)}{\partial x^{2}}\right]^{2} d x=\frac{1}{2} \frac{L^{3} M_{t} g}{E I \bar{\mu}(L) \phi(L)} \cdot \frac{E I}{L^{3}} w^{2}(L),
\end{gathered}
$$

where $w(L)$ and $\dot{w}(L)$ are the tip displacement and the tip velocity of the PVEH, respectively. 
TABLE 1: Theoretical and fitting results of $\mu(x) \times 10^{-5}$.

\begin{tabular}{lccccc}
\hline Length/m & 0.01 & 0.02 & 0.03 & 0.04 & 0.05 \\
Theoretical results & -1.2121 & -1.2182 & -1.2235 & -1.2292 & -1.2338 \\
Fitting results & -1.2118 & -1.2181 & -1.2237 & -1.2294 & -1.2343 \\
\hline
\end{tabular}

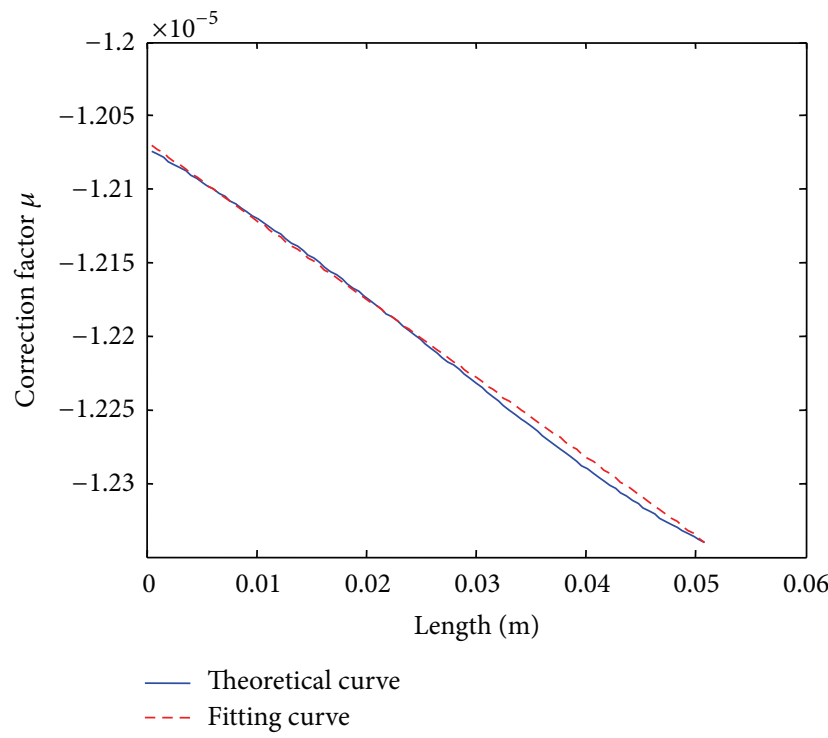

FIGURE 3: Correction factor with cantilevered length for theoretical calculation and fitting results.

The corresponding kinetic energy and potential energy of the PVEH without tip mass calculated with the ULPM are described by [10]

$$
\begin{gathered}
T^{\prime}=\frac{1}{2} m_{\mathrm{eq}} d x \dot{w}^{2}(L), \\
E_{u}^{\prime}=\frac{1}{2} K w^{2}(L),
\end{gathered}
$$

where $m_{\mathrm{eq}}$ and $K$ are the equivalent mass and the equivalent stiffness, respectively, of the PVEH without tip mass. According to the Rayleigh-Ritz approximation, the kinetic energy and the potential energy of the PVEH must be conserved, and we therefore have

$$
T=T^{\prime}, \quad E_{u}=E_{u}^{\prime} .
$$

Therefore, $m_{\mathrm{eq}}$ and $K$ can be obtained from (20) as follows:

$$
\begin{gathered}
m_{\mathrm{eq}}=\int_{0}^{L} \rho A\left[\frac{\bar{\mu}(x) \phi(x)}{\bar{\mu}(L) \phi(L)}\right]^{2} d x, \\
K=\frac{L^{3} M_{t} g}{\operatorname{EI\mu }(L) \phi(L)} \cdot \frac{E I}{L^{3}} .
\end{gathered}
$$

Comparing (21) with the first term of the right-hand side expression of (11), the correction factor $\beta_{M}$ of the equivalent mass $M$ is

$$
\beta_{M}=\frac{\int_{0}^{L}[\bar{\mu}(x) \phi(x)]^{2} d x}{[\bar{\mu}(L) \phi(L)]^{2} L} .
$$

TABLE 2: Material and geometric parameters. Source: Erturk and Inman, 2009 [15].

\begin{tabular}{lc}
\hline Item & Value \\
\hline Piezoelectric plate density $\rho_{p}\left(\mathrm{~kg} / \mathrm{m}^{3}\right)$ & 7800 \\
Substrate plate density $\rho_{s}\left(\mathrm{~kg} / \mathrm{m}^{3}\right)$ & 9000 \\
Piezoelectric plate stiffness $E_{p}(\mathrm{GPa})$ & 66 \\
Substrate plate stiffness $E_{s}(\mathrm{GPa})$ & 105 \\
Strain constant $d_{31}(\mathrm{C} / \mathrm{m})$ & $-190 \times 10^{-12}$ \\
Stress constant $e_{31}(\mathrm{C} / \mathrm{m})$ & -11.5 \\
Vacuum permittivity $\varepsilon_{0}(\mathrm{~F} / \mathrm{m})$ & $8.854 \times 10^{-12}$ \\
Absolute permittivity $\varepsilon_{33}^{S}(\mathrm{~F} / \mathrm{m})$ & $1500 \varepsilon_{0}$ \\
Beam length $L(\mathrm{~mm})$ & 50.8 \\
Beam width $b(\mathrm{~mm})$ & 31.8 \\
Piezoelectric plate thickness $h_{p}(\mathrm{~mm})$ & 0.26 \\
Substrate platethickness $h_{s}(\mathrm{~mm})$ & 0.14 \\
Damping ratio of elastic magnifier $\xi_{b}$ & 0.002 \\
Damping ratio of piezoelectric beam $\xi_{\mathrm{eq}}$ & 0.027 \\
Tip mass $M_{t}(\mathrm{~kg})$ & 0.012 \\
Load resistance $R_{L}(\Omega)$ & 1000 \\
\hline
\end{tabular}

Similarly, comparing (22) with (12), the correction factor $\beta_{K}$ of the equivalent stiffness $K$ is

$$
\beta_{K}=\frac{L^{3} M_{t} g}{\operatorname{EI\mu }(L) \phi(L)} .
$$

The electromechanical coupling effects of the PZTs are identified by the factor $\alpha$ and electrical capacitance $C_{p}$, and these may be defined according to the well-known piezoelectric constitutive equation and the relationships between the stress and the strain of the PVEH, respectively [25]:

$$
S=s^{E} T+d E, \quad D=d T+\varepsilon^{T} E,
$$

where $S$ is the strain, $T$ is the stress, $D$ is the electric displacement, $E$ is the electric field, $d$ is the piezoelectric constant, $s^{E}$ is the mechanical compliance at constant electric field, and $\varepsilon^{T}$ is the permittivity at constant stress:

$$
\begin{gathered}
\alpha=\beta_{\alpha} \frac{b\left(h_{s}+h_{p}\right)}{2 L} e_{31}, \\
C_{p}=\frac{b L}{2 h_{p}} \varepsilon_{33}^{S},
\end{gathered}
$$

where $\beta_{\alpha}=-\left.L(d \phi(x) / d x)\right|_{x=L}$ and $e_{31}$ and $\varepsilon_{33}^{S}$ are the piezoelectric and clamped dielectric constants, respectively.

Therefore, we can obtain the resonance and the antiresonance frequencies of the PVEH from (11), (12), and (26), respectively [26]:

$$
f_{r}=\frac{\sqrt{K / M}}{(2 \pi)}, \quad f_{a r}=\sqrt{1+d^{2}} f_{r},
$$

where $d^{2}=\alpha^{2} /\left(K C_{p}\right)$. 
TABLE 3: Frequencies comparison of ILPM with LPM, ULPM, and CDPM.

\begin{tabular}{lcc}
\hline Models & $\begin{array}{c}\text { Resonance } \\
\text { frequency }(\mathrm{Hz})\end{array}$ & $\begin{array}{c}\text { Antiresonance } \\
\text { frequency }(\mathrm{Hz})\end{array}$ \\
\hline Numerical results of ILPM & 45.68 & 48.91 \\
Theoretical results of CDPM & 45.7 & 48.2 \\
Experimental results of CDPM & 45.6 & 48.4 \\
Theoretical result of LPM & 45.65 & \\
Theoretical result of ULPM & 44.401 & \\
\hline
\end{tabular}

\section{Numerical Results}

Here we describe a numerical calculation used to analyze the performance of the PVEH with MATLAB software. Table 2 lists the material and geometric parameters of the PVEH.

Putting the material and geometric parameters from Table 2 into (23), (24), and (26), we can obtain the equivalent parameters of the PVEH. By then putting these equivalent parameters into (5)-(7), we can obtain the output performances of the PVEH in transverse vibration.

Figure 4 shows the relations between the resonance frequency of the PVEH and the mass ratio $a$ (where $a=$ $M_{t} / m_{\mathrm{eq}}$ ). It can be seen from Figure 4 that the resonance frequency of the PVEH decreases with increasing mass ratio, which means that increasing the tip mass $M_{t}$ lowers the resonance frequency to facilitate approaching ambient vibration frequency.

Figures 5,6 , and 7 show the velocity response $\dot{x} / \ddot{y}$ of the tip mass, the output voltage response $V / \ddot{y}$, and the output power response $P / \ddot{y}$ of the load resistance, respectively, with various mass ratios $a$ when the load resistance $R_{L}=$ $1 \mathrm{k} \Omega$. A semilogarithmic coordinate is used for displaying the difference of each power output curve more clearly in Figure 7. As the mass ratio $a$ increases, the tip velocity, the output voltage, and the output power of the PVEH all increase, the maximum values (or peaks) of each curve can be achieved at the corresponding resonant conditions, and the peaks of the curves shift to the left with increasing mass ratio $a$. Therefore, the resonance frequency of the PVEH can be decreased by increasing the mass ratio. This conclusion is consistent with the results shown in Figure 4. Moreover, when the mass ratio $a=10$, the tip velocity, the output voltage, and the output power are approximately $0.8((\mathrm{~m} / \mathrm{s}) / \mathrm{g}), 2.1$ $(\mathrm{V} / \mathrm{g})$, and $4.4(\mathrm{~mW} / \mathrm{g})$, respectively, that is, larger than the respective values when $a=0$. The analytical results show that a large tip mass $M_{t}$ can lower the resonance frequency and improve the output performances of, for example, tip velocity, output voltage, and output power.

Figure 8 shows simulation curves of the output voltage and the tip velocity of the PVEH for various values of load resistance. The direction of increasing load resistance is depicted by an arrow. It is clear from Figure 8(a) that the voltage across the resistive load increases monotonically with increasing load resistance at every excitation frequency. For extreme values of load resistance, the frequency of the peak voltage moves from the short-circuit resonance frequency (for $R_{L} \rightarrow 0$ ) to the open-circuit resonance frequency

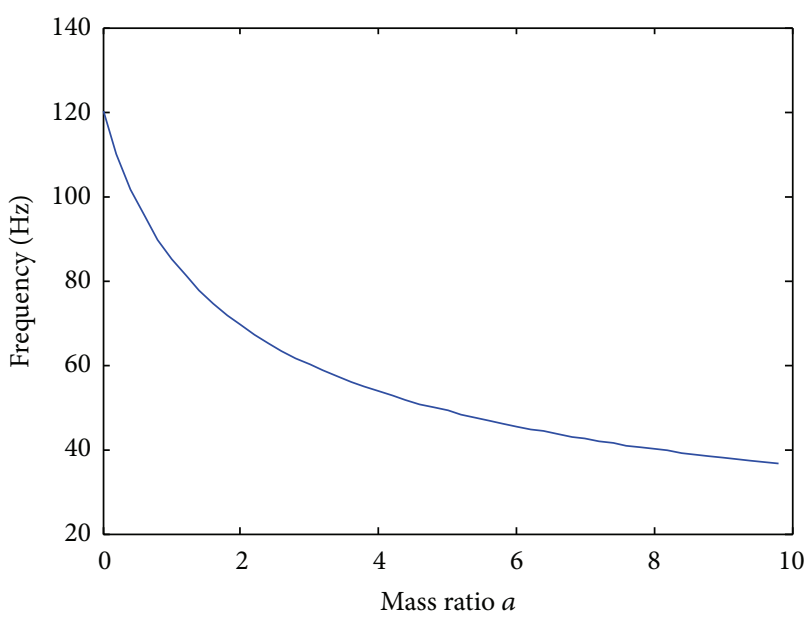

FIGURE 4: Variation in the resonance frequency of the PVEH with various mass ratios $a$.

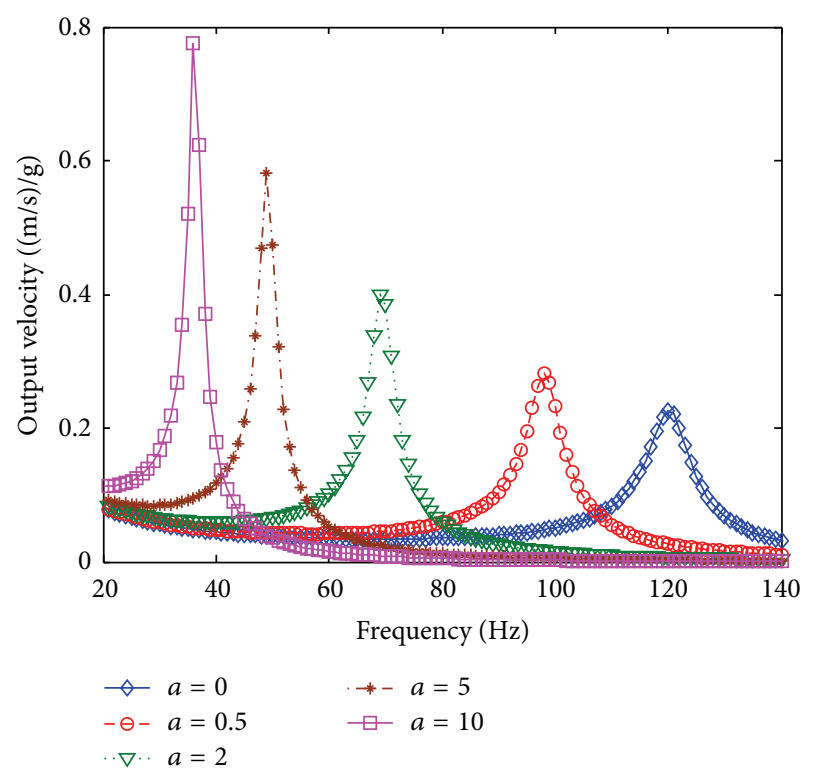

FIGURE 5: Output velocity for the PVEH with various mass ratios $a$ when $R_{L}=1 \mathrm{k} \Omega$.

(for $R_{L} \rightarrow \infty$ ). The analytical model predicts these two frequencies as $45.68 \mathrm{~Hz}$ and $48.91 \mathrm{~Hz}$, respectively. For a moderate load resistance, the frequency of the peak voltage of the PVEH is between these two extreme frequencies.

Figure 8(b) shows the shift in the frequencies of peaks in response amplitude of the tip velocity of the PVEH for various load resistances. Variation of the tip velocity of the PVEH with load resistance is not monotonic at each frequency. For example, when excitation occurs at $45.68 \mathrm{~Hz}$, the tip velocity decreases as the load resistance increases to a particular value. However, it increases as the load resistance increases when excitation occurs at $48.91 \mathrm{~Hz}$.

Figure 9 shows the output current and the output power of the PVEH for various load resistances. The output current (obtained from $I=V / R_{L}$ ) exhibits the opposite behavior to the output voltage with changing load resistance, as shown in 


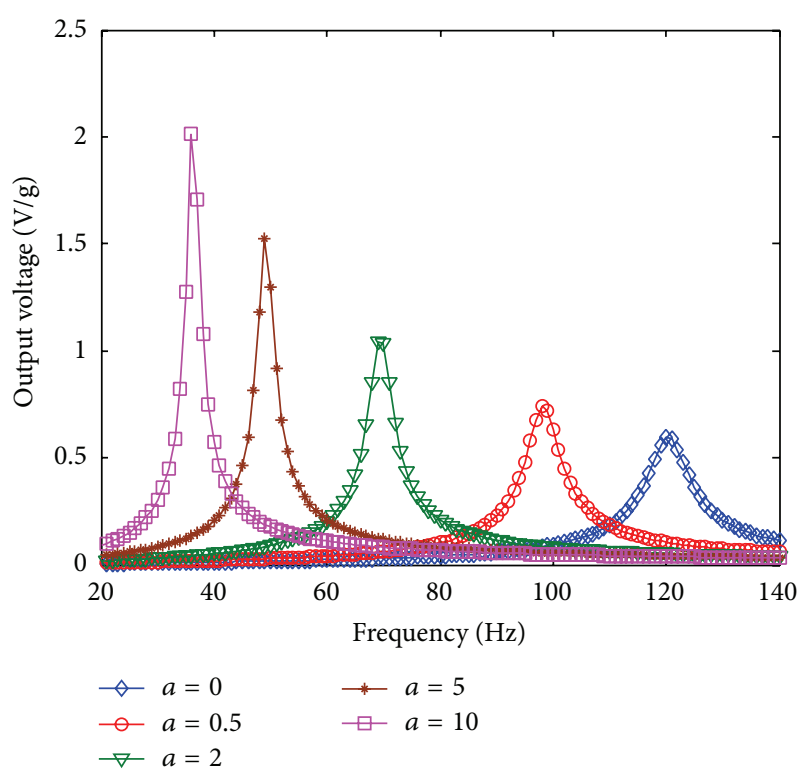

FIGURE 6: Output voltage for the PVEH with various mass ratios $a$ when $R_{L}=1 \mathrm{k} \Omega$.

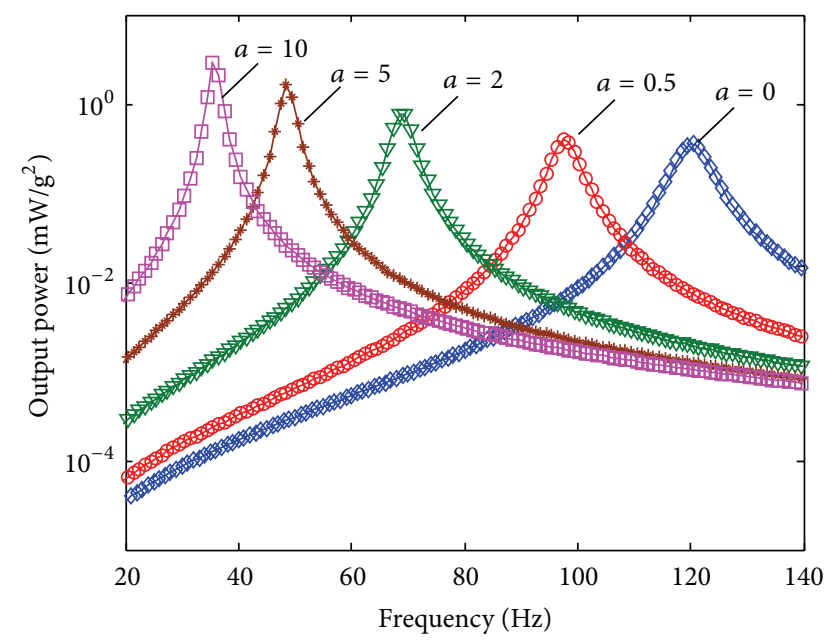

FIGURE 7: Output power for the PVEH with various mass ratios $a$ when $R_{L}=1 \mathrm{k} \Omega$.

Figure 9(a). This indicates that the current flowing into the resistive load decreases monotonically with increasing load resistance at every excitation frequency. As can be seen from Figure 9(b), the output power of the PVEH for various load resistances intersects like the tip velocity (Figure 8(b)). For a given excitation frequency, there is a particular value of load resistance at which the maximum output power can be realized.

The maximum output power of the PVEH can be obtained at resonance frequency and antiresonance frequency for the short-circuit condition $\left(R_{L} \rightarrow 0\right)$ and the open-circuit condition $\left(R_{L} \rightarrow \infty\right)$, respectively. By using (27) for the natural frequencies, the resonance frequency and antiresonance frequency are calculated as $45.68 \mathrm{~Hz}$ and $48.91 \mathrm{~Hz}$, respectively. Variation of the output voltage with load resistance for excitation at these two frequencies is shown in Figure 10(a). In both curves, the voltage increases monotonically with load resistance. The output voltage for excitation at short-circuit resonance frequency is larger than that of the system close to the open-circuit condition. The maximum voltage amplitude is approximately $49.5 \mathrm{~V} / \mathrm{g}$ for excitation at $45.68 \mathrm{~Hz}$ and is approximately $129.3 \mathrm{~V} / \mathrm{g}$ for excitation at $48.91 \mathrm{~Hz}$. Figure $10(\mathrm{~b})$ shows variation of the output current with load resistance for excitation at these two frequencies. The trend of output current with load resistance is opposite to that of output voltage. The output current decreases monotonically with increasing load resistance. The maximum current amplitude is approximately $1.65 \mathrm{~mA} / \mathrm{g}$ for excitation at $45.68 \mathrm{~Hz}$ and is approximately $0.63 \mathrm{~mA} / \mathrm{g}$ for excitation at $48.91 \mathrm{~Hz}$. Variation of the output power with load resistance for excitation at these two frequencies is shown in Figure 10(c). An optimum load resistance appears to cause the output power to achieve the maximum value for each excitation frequency. The optimum load resistance for excitation at $45.68 \mathrm{~Hz}$ is approximately $30 \mathrm{k} \Omega$, yielding a maximum output power of approximately $21.92 \mathrm{~mW} / \mathrm{g}^{2}$, whereas the optimum load resistance for excitation at $48.91 \mathrm{~Hz}$ is approximately $215 \mathrm{k} \Omega$, yielding a maximum output power of approximately $21.85 \mathrm{~mW} / \mathrm{g}^{2}$.

Figure 11 shows variation in the output power with excitation frequencies for these two optimum load resistances. Note that the peak power generated at the optimum load resistance of $215 \mathrm{k} \Omega$ is close to that generated at the optimum load resistance of $30 \mathrm{k} \Omega$. Comparing Figure 11 with Figure 10, we find that matching load resistances under the resonance and antiresonance frequency conditions produces almost the same maximum power, although they produce different maximum output voltage and current.

\section{Model Verification}

The verified analytical results are compared with the research results obtained from the ULPM, the LPM, and the CDPM derived under the Euler-Bernoulli beam assumptions [13-15].

Using (23) and (24), the equivalent parameters of the ILPM can be calculated as

$$
\beta_{M}=0.2355, \quad \beta_{K}=2.9889 .
$$

The fundamental natural frequency (or resonance frequency) calculated with the equivalent parameters of (28) is $45.68 \mathrm{~Hz}$, where the error in predicting the fundamental natural frequency of the PVEH is approximately $0.044 \%$ relative to the CDPM model fundamental natural frequency.

Table 3 lists the resonance frequency and the antiresonance frequency calculated by the LPM, the ULPM, the CDPM, and the ILPM proposed in this paper. The data in Table 3 indicate that the numerical results of the ILPM are in good agreement with the analytical results of the CDPM. The results listed in Table 3 verify that prediction of the fundamental natural frequency through the ILPM may be improved.

Figures 12 and 13 show the results of comparison of the tip velocity, the output voltage, and the output power obtained 


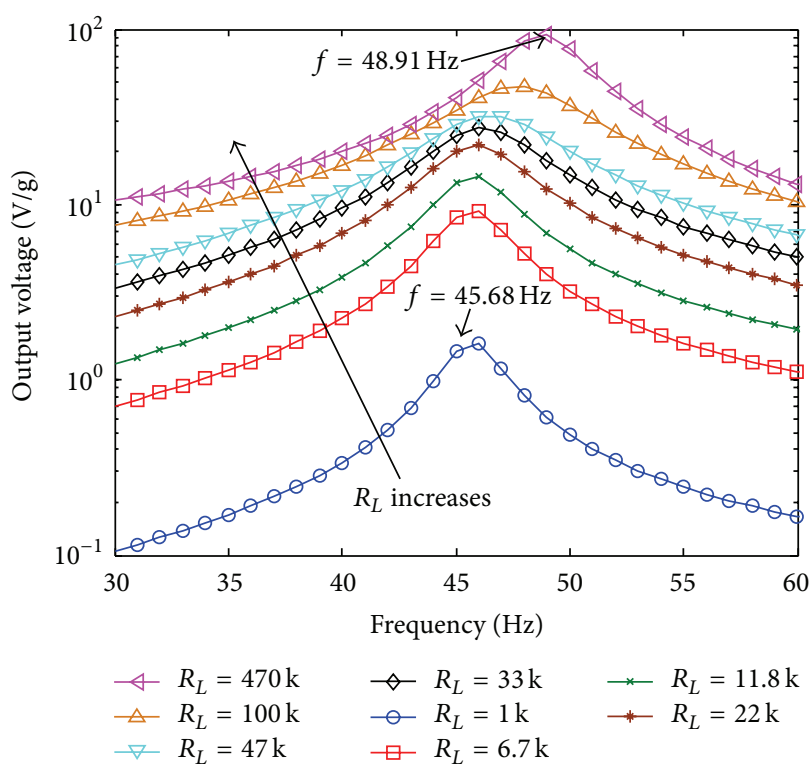

(a)

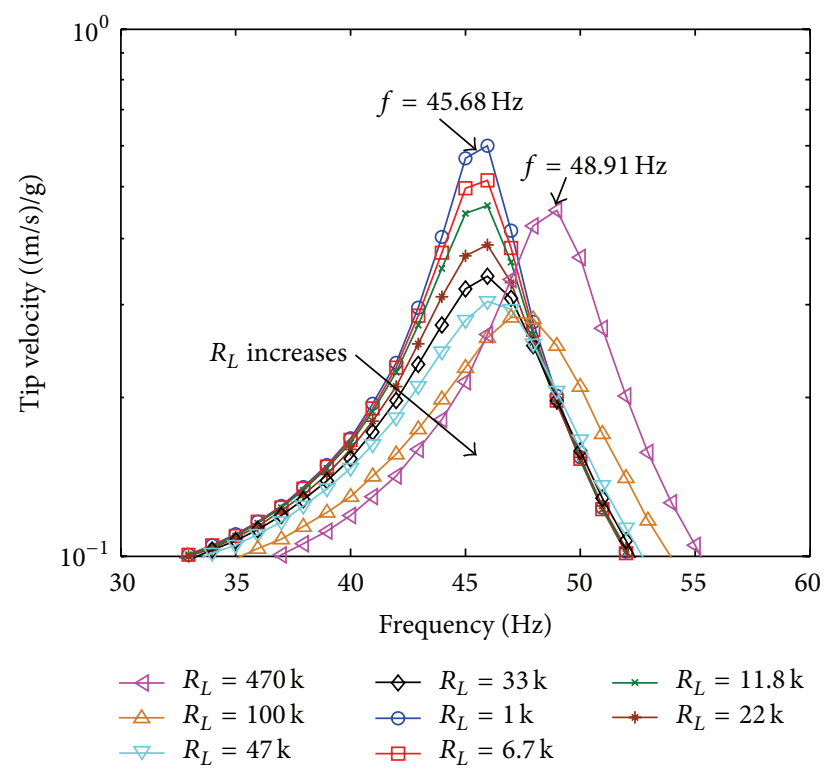

(b)

FIgURE 8: Output voltage (a) and tip velocity (b) of the PVEH for various values of load resistance.

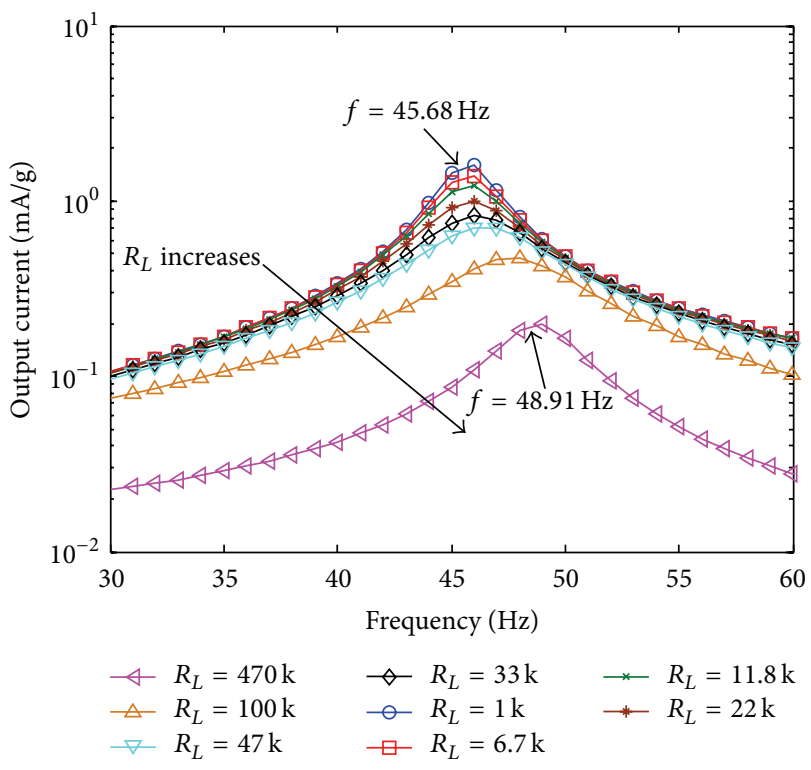

(a)

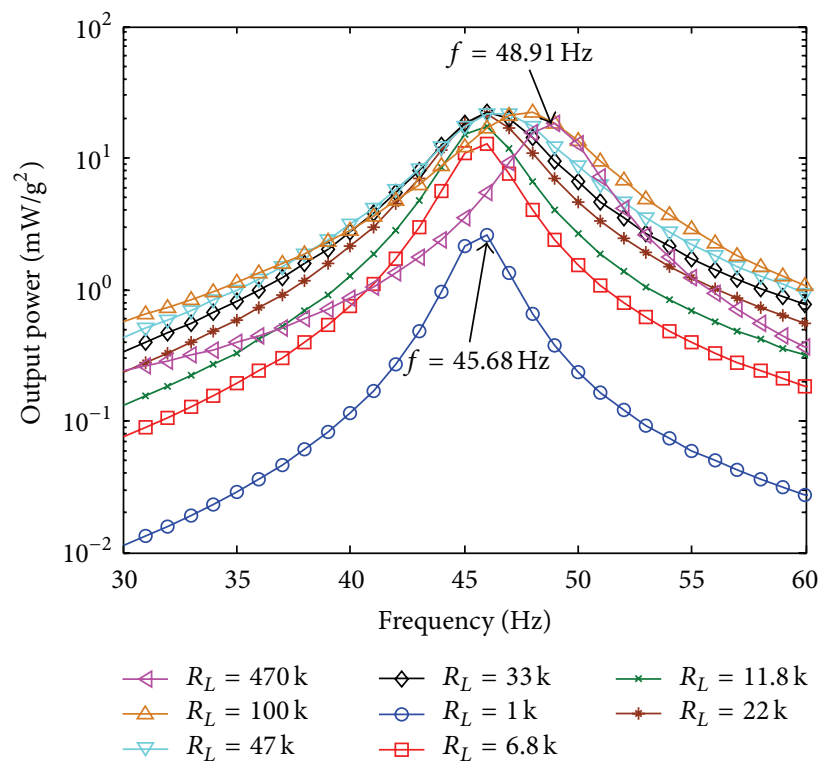

(b)

FIGURE 9: Output current (a) and output power (b) of the PVEH for various values of load resistance.

from the ULPM, the LPM, the ILPM, and the CDPM under various excitation frequencies when $R_{L}=1 \mathrm{k} \Omega$ (Figure 12) and $R_{L}=470 \mathrm{k} \Omega$ (Figure 13). Because the ULPM does not take into account the coupling effects of the PZTs, it can be used to predict only the tip velocity of the PVEH, and the output voltage and output power of the PVEH cannot be predicted.
As can be seen from the results in these figures, the tip velocity, the output voltage, and the output power of the ILPM are in perfect agreement with the results obtained from the LPM and the CDPM. This clearly indicates the validity of the proposed ILPM.

In addition, when the resistive load is increased to $470 \mathrm{k} \Omega$ (Figure 13), the resonance frequency shifts from 45.68 to 


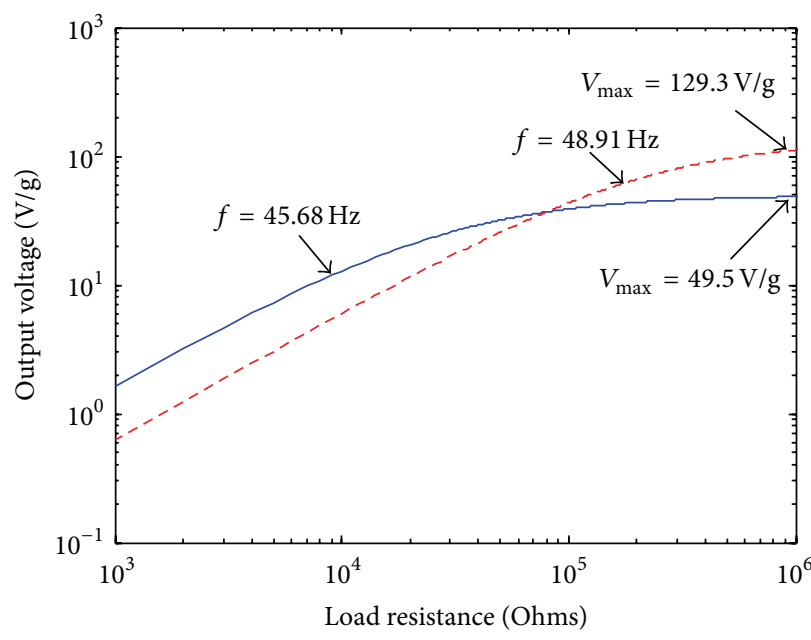

(a)

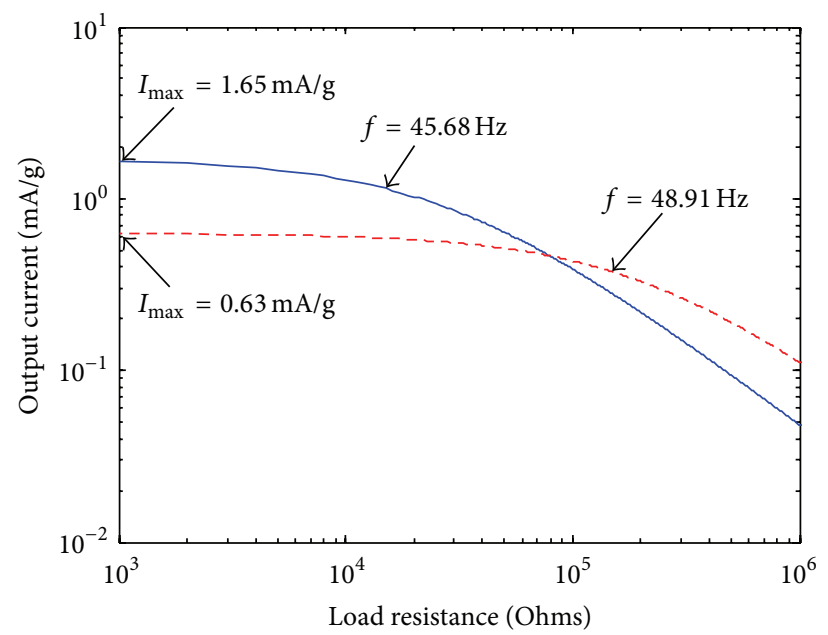

(b)

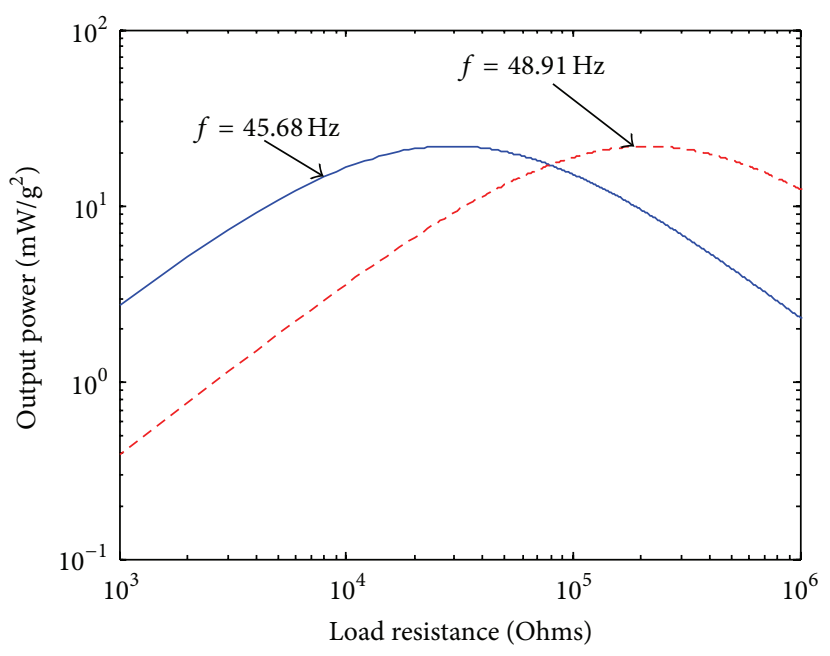

(c)

FIGURE 10: Variations in output voltage, output current, and output power of PVEH with load resistance at the resonance and antiresonance frequencies: (a) voltage, (b) current, and (c) power.

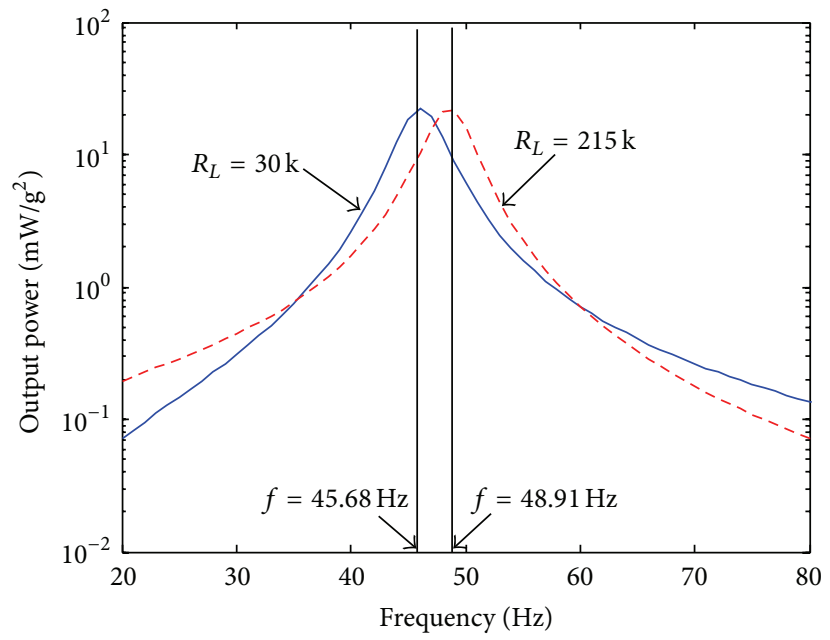

FIGURE 11: Variation in output power of the PVEH with excitation frequencies for two optimum load resistances. 


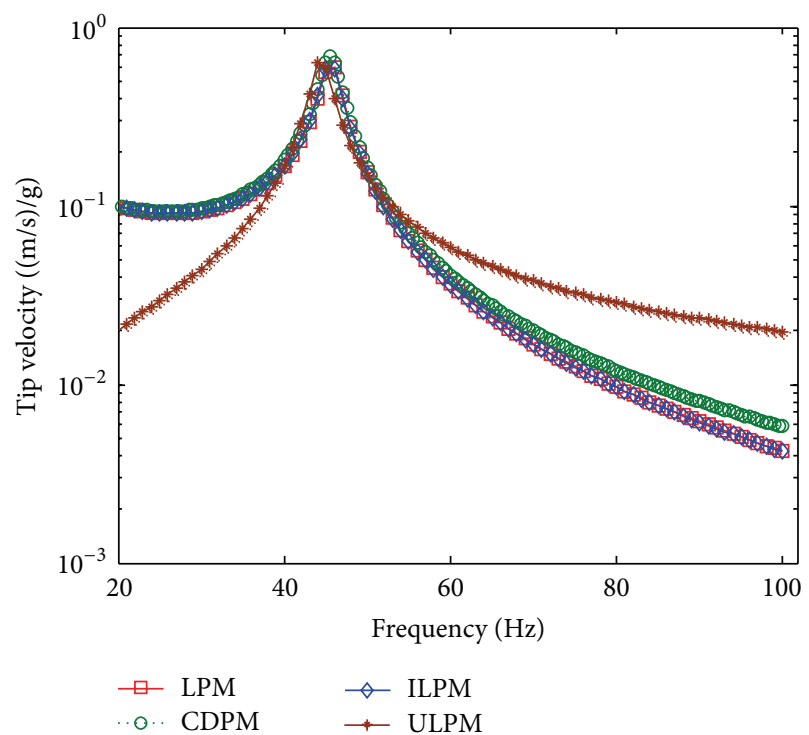

(a)

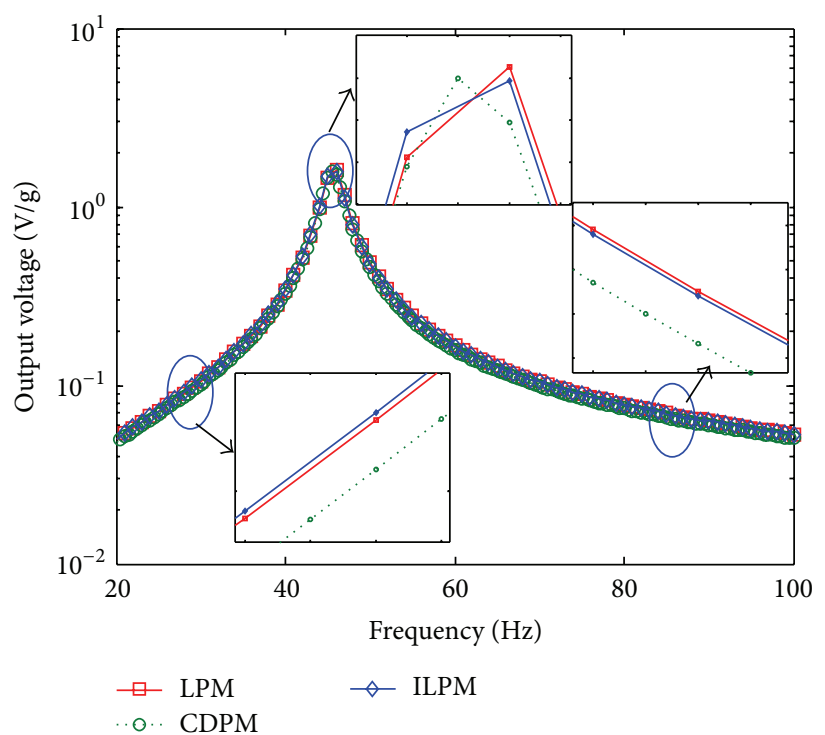

(b)

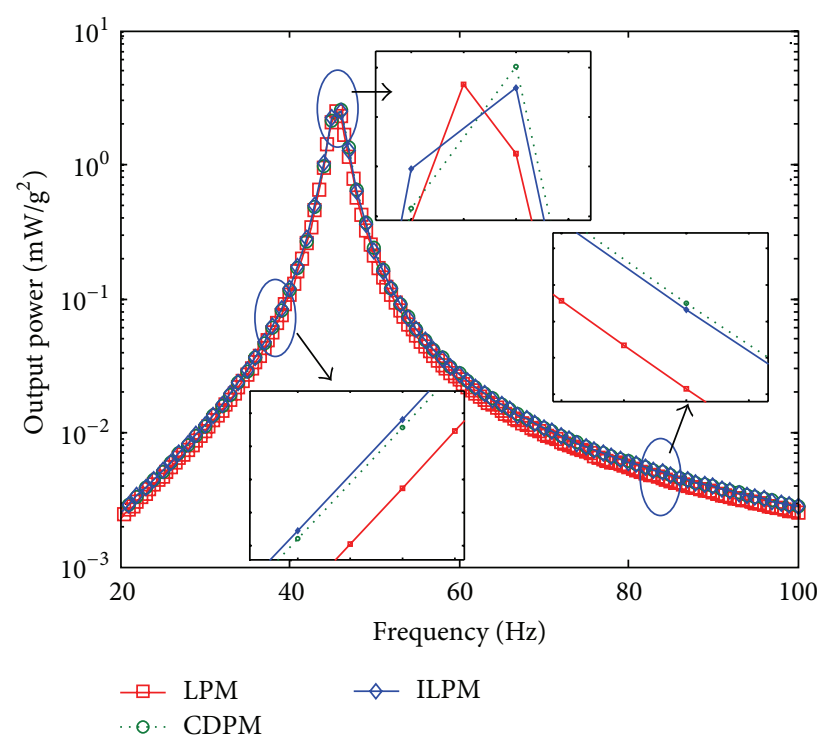

(c)

FIGURE 12: Comparison of analytical results of LPM, CDPM, ULPM, and ILPM when $R_{L}=1 \mathrm{k} \Omega$ : (a) tip velocity, (b) output voltage, and (c) output power.

$48.91 \mathrm{~Hz}$, which is approximately $3.22 \mathrm{~Hz}$ higher than the resonance frequency for $1 \mathrm{k} \Omega$ (Figure 12). Variations in the resonance frequency with changing load resistance, and the amplitude-wise results, are successfully predicted by the ILPM.

\section{Conclusions}

We have proposed an ILPM of the PVEH that takes into account dynamic mode and accurate strain distribution. The results obtained from the ILPM were in good agreement with those from the LPM and the CDPM. The proposed
ILPM successfully predicted the amplitude-wise results and the variations in resonance frequency with changing load resistance.

The results demonstrate the feasibility of the proposed ILPM as a simple and effective means of predicting the output performances of the PVEH. Currently, the ILPM is taken to be identical to the first natural frequency of the CDPM, and the ILPM is extended to harvesters that rely on operating cantilevered beams under a clamped-free boundary condition. Further work is in progress to reduce the error of the ILPM at higher frequencies and to explain the effect of various boundary conditions on output performances of the PVEH. 


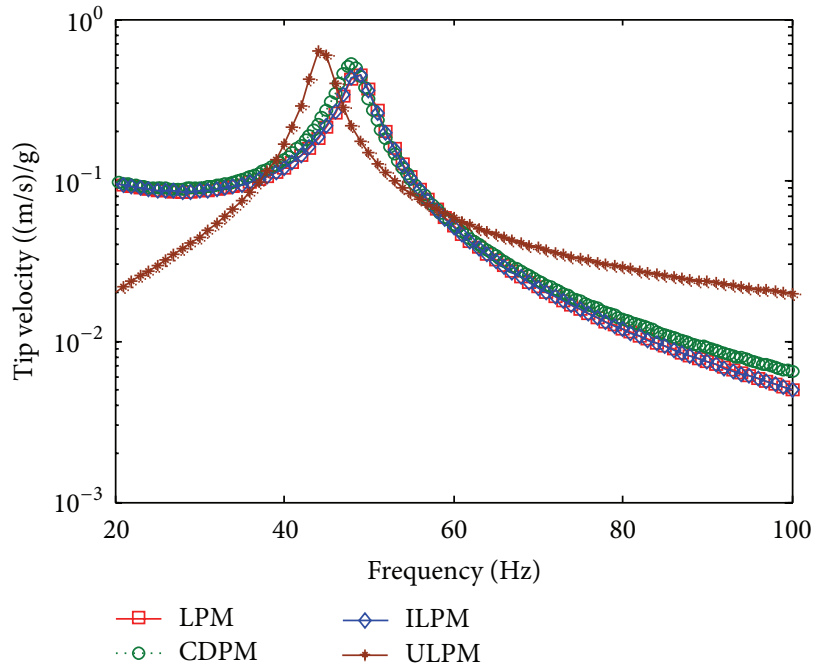

(a)

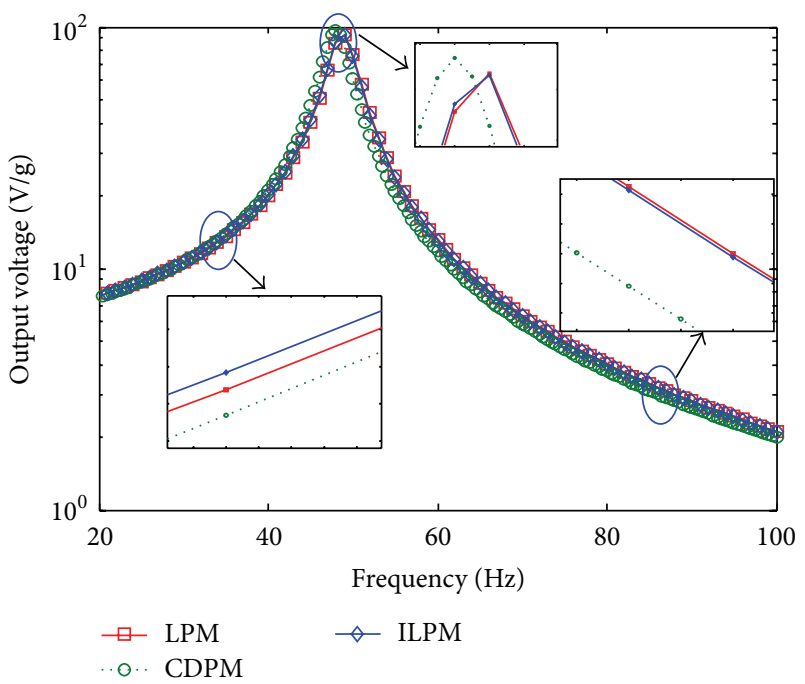

(b)

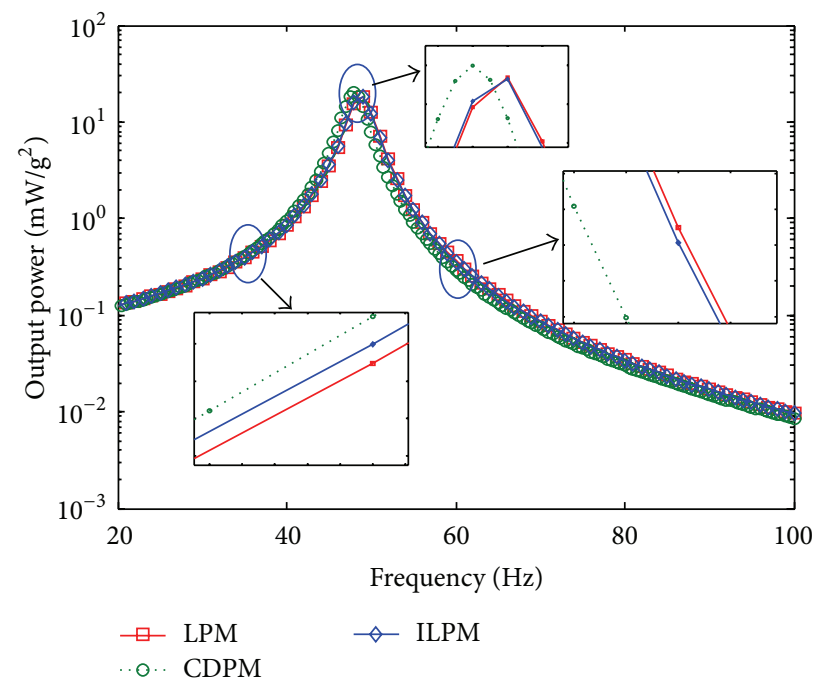

(c)

FIGURE 13: Comparison of analytical results of LPM, CDPM, ULPM, and ILPM when $R_{L}=470 \mathrm{k} \Omega$ : (a) tip velocity, (b) output voltage, and (c) output power.

\section{Conflict of Interests}

The authors declare that there is no conflict of interests regarding the publication of this paper.

\section{Acknowledgments}

This project was supported by the National Natural Science Foundation of China (no. 51277165), the Foundation of Zhejiang Educational Committee (no. Y201223050), the Science Foundation for the University Excellent Youth Scholars of Zhejiang Province, and the Science Foundation for the Youth Talents of Zhejiang Gongshang University (no. QY11-23).

\section{References}

[1] J. Ajitsaria, S. Y. Choe, D. Shen, and D. J. Kim, "Modeling and analysis of a bimorph piezoelectric cantilever beam for voltage generation," Smart Materials and Structures, vol. 16, no. 2, pp. 447-454, 2007.

[2] S. R. Anton and H. A. Sodano, "A review of power harvesting using piezoelectric materials (2003-2006)," Smart Materials and Structures, vol. 16, no. 3, pp. R1-R2, 2007.

[3] N. S. Hudak and G. G. Amatucci, "Small-scale energy harvesting through thermoelectric, vibration, and radiofrequency power conversion," Journal of Applied Physics, vol. 103, no. 10, Article ID 101301, 2008.

[4] C. O. Mathuna, T. O'Donnel, R. V. Martinez-Catala, J. Rohan, and B. O’Flynn, “Energy scavenging for long-term deployable 
wireless sensor networks," Talanta, vol. 75, no. 3, pp. 613-623, 2008.

[5] J. A. Paradiso and T. Starner, "Energy scavenging for mobile and wireless electronics," IEEE Pervasive Computing, vol. 4, no. 1, pp. 18-27, 2005.

[6] J. M. Rabaey, M. J. Ammer, J. L. Da Silva Jr., D. Patel, and S. Roundy, "Picoradio supports ad hoc ultra-low power wireless networking," Computer, vol. 33, no. 7, pp. 42-48, 2000.

[7] S. P. Beeby, M. J. Tudor, and N. M. White, "Energy harvesting vibration sources for microsystems applications," Measurement Science and Technology, vol. 17, no. 12, pp. R175-R195, 2006.

[8] J.-B. Yuan, T. Xie, X.-B. Shan, and W.-S. Chen, "Resonant frequencies of a piezoelectric drum transducer," Journal of Zhejiang University A, vol. 10, no. 9, pp. 1313-1319, 2009.

[9] S. Roundy, P. K. Wright, and J. Rabaey, "A study of low level vibrations as a power source for wireless sensor nodes," Computer Communications, vol. 26, no. 11, pp. 1131-1144, 2003.

[10] C. B. Williams and R. B. Yates, "Analysis of a micro-electric generator for microsystems," Sensors and Actuators A, vol. 52, no. 1-3, pp. 8-11, 1996.

[11] N. W. Hagood, W. H. Chung, and A. Von Flotow, "Modeling of piezoelectric actuator dynamics for active structural control," Journal of Intelligent Material Systems and Structures, vol. 1, no. 3, pp. 327-354, 1990.

[12] H. A. Sodano, G. Park, and D. J. Inman, "Estimation of electric charge output for piezoelectric energy harvesting," Strain, vol. 40, no. 2, pp. 49-58, 2004.

[13] A. Erturk and D. J. Inman, "On mechanical modeling of cantilevered piezoelectric vibration energy harvesters," Journal of Intelligent Material Systems and Structures, vol. 19, no. 11, pp. 1311-1325, 2008.

[14] A. Erturk and D. J. Inman, "A distributed parameter electromechanical model for cantilevered piezoelectric energy harvesters," ASME Journal of Vibration and Acoustics, vol. 130, no. 4, Article ID 041002, 2008.

[15] A. Erturk and D. J. Inman, "An experimentally validated bimorph cantilever model for piezoelectric energy harvesting from base excitations," Smart Materials and Structures, vol. 18, no. 2, Article ID 025009, 2009.

[16] H. Y. Wang, X. T. Shan, and T. Xie, "An energy harvester combining a piezoelectric cantilever and a single degree of elastic system," Journal of Zhejiang University A, vol. 13, no. 7, article 526, 2012.

[17] N. E. duToit, B. L. Wardle, and S. G. Kim, "Design considerations for MEMS-scale piezoelectric mechanical vibration energy harvesters," Integrated Ferroelectrics, vol. 71, no. 1, pp. 121-160, 2005.

[18] Y. Liao and H. A. Sodano, "Model of a single mode energy harvester and properties for optimal power generation," Smart Materials and Structures, vol. 17, no. 6, Article ID 065026, 2008.

[19] N. G. Stephen, "On energy harvesting from ambient vibration," Journal of Sound and Vibration, vol. 293, no. 1-2, pp. 409-425, 2006.

[20] Y. B. Jeon, R. Sood, J.-H. Jeong, and S.-G. Kim, "MEMS power generator with transverse mode thin film PZT," Sensors and Actuators A, vol. 122, no. 1, pp. 16-22, 2005.

[21] C. W. Lim and L. H. He, "Three-dimensional exact solutions for the electromechanical response of triple-layer piezoelectric actuators," Smart Materials and Structures, vol. 13, no. 5, pp. 1050-1058, 2004.
[22] J. W. Strutt (Lord Rayleigh), The Theory of Sound, MacMillan Company, London, UK, 1984.

[23] S. Timoshenko, D. H. Young, and W. Weaver, Vibration Problems in Engineering, John Wiley \& Sons, New York, NY, USA, 1974.

[24] S. J. Ahn, Least Squares Orthogonal Distance Fitting of Curves and Surfaces, vol. 3151 of Lecture Notes in Computer Science, Springer, 2004.

[25] IEEE, IEEE Standard on Piezoelectricity, ANSI/IEEE Std 1761987, New York, NY, USA, 1987.

[26] Y. C. Shu and I. C. Lien, "Analysis of power output for piezoelectric energy harvesting systems," Smart Materials and Structures, vol. 15, no. 6, pp. 1499-1512, 2006. 

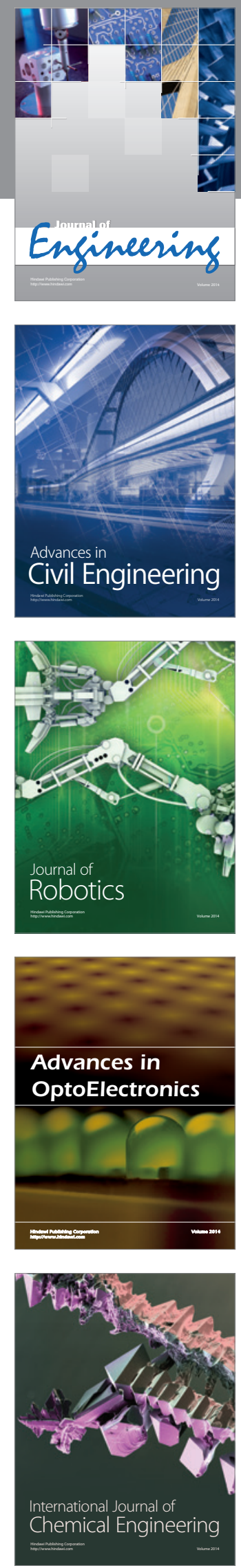

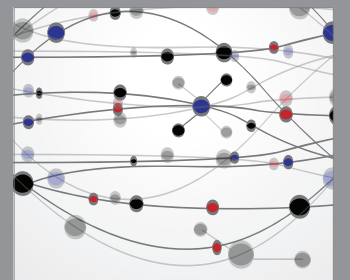

The Scientific World Journal
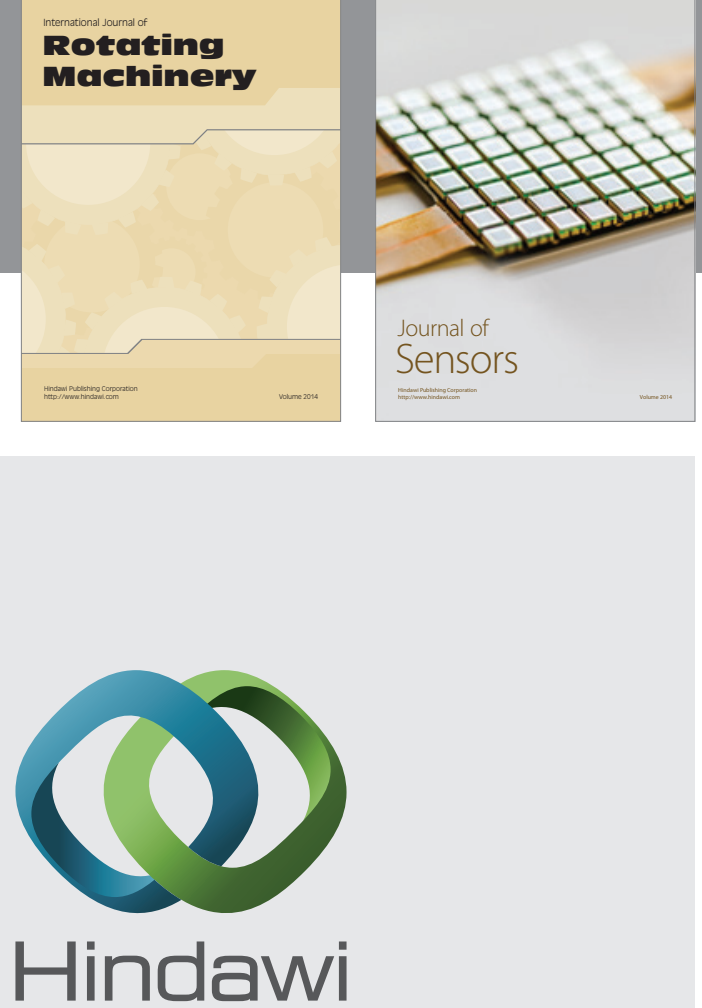

Submit your manuscripts at http://www.hindawi.com
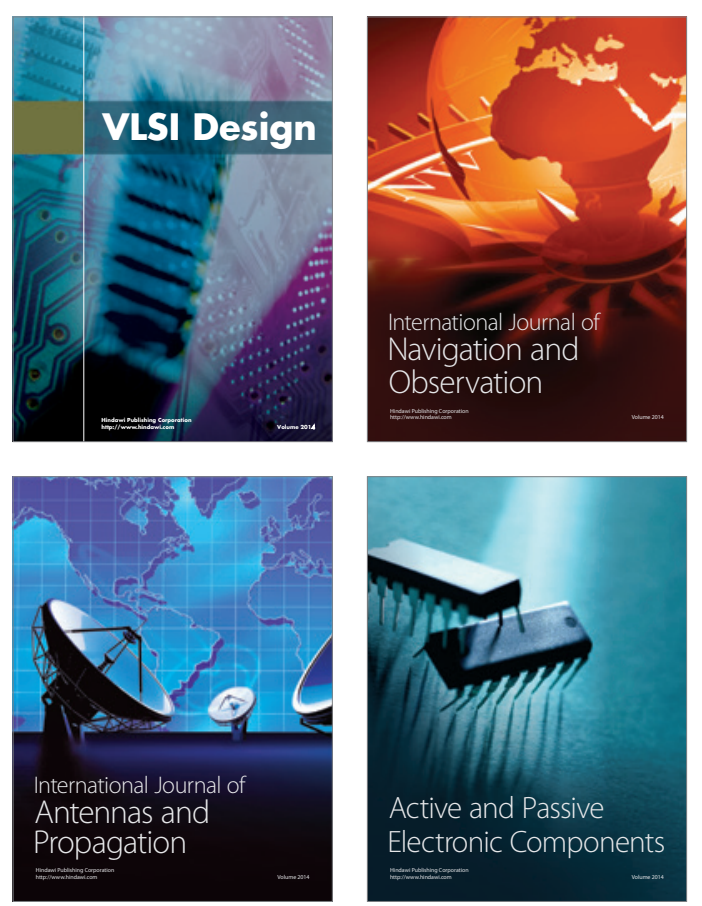
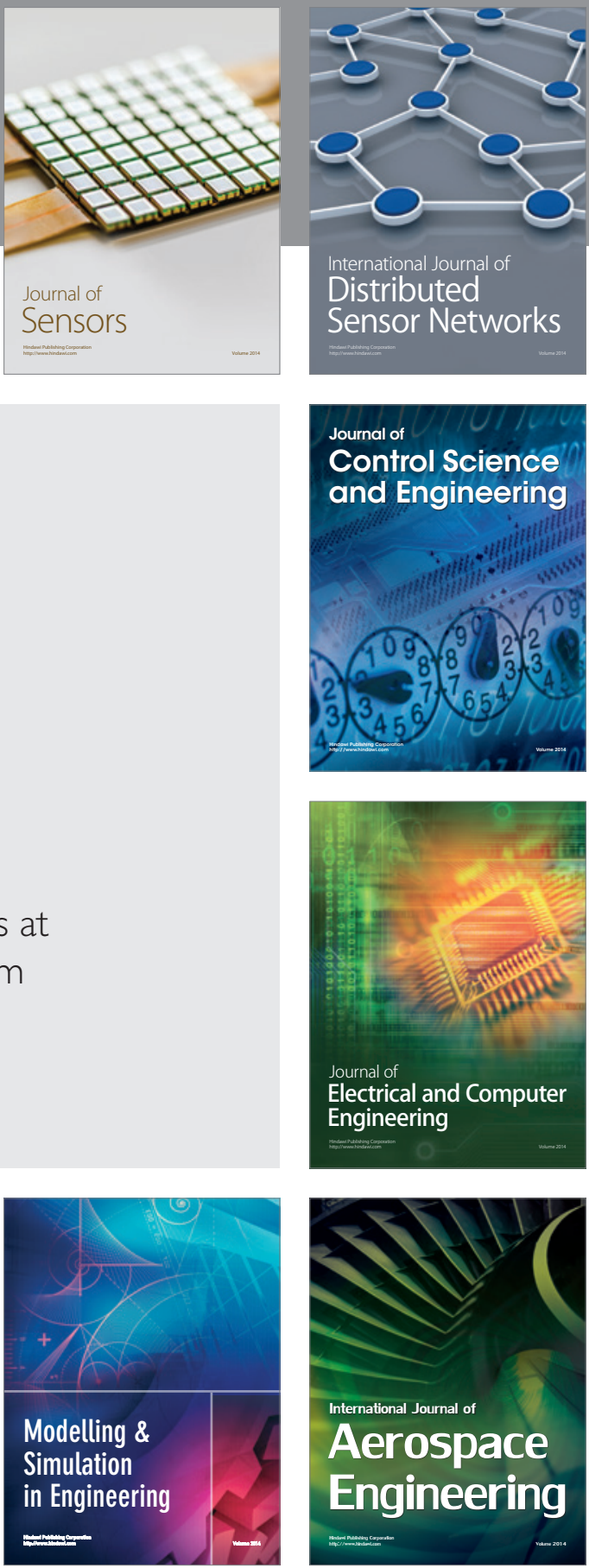

Journal of

Control Science

and Engineering
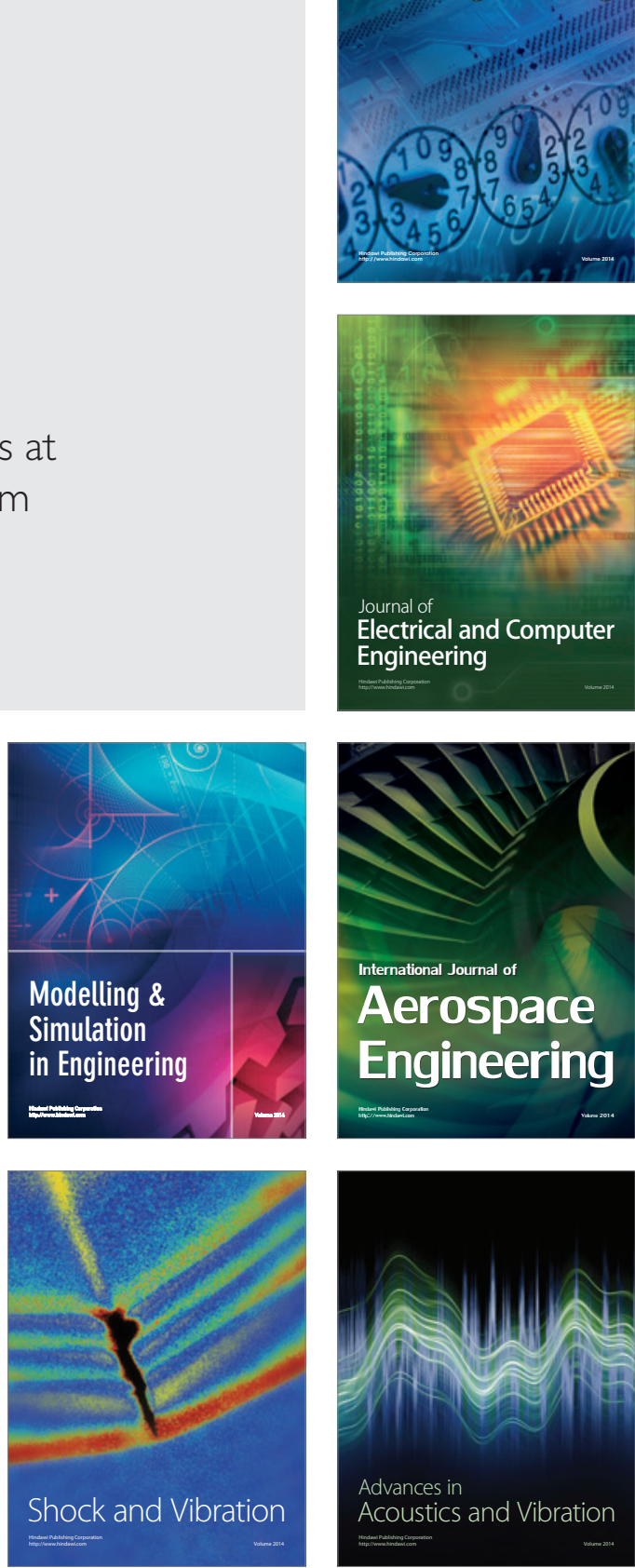